\title{
Mapping and Controlling Strain in Epitaxially Connected Quantum Dot Superlattices - a Path to Designer Quantum Materials
}

\author{
Michelle Smeaton, Ismail El Baggari, Daniel Balazs, Tobias Hanrath and Lena Kourkoutis \\ Cornell University, Ithaca, New York, United States
}

Epitaxially connected superlattices of quantum dots, called quantum dot solids (QDS), have been identified as a versatile system for studying exotic quantum phenomena such as Dirac cones, spin-orbit coupling and topological states [1]. Such electronic features are theoretically attainable due to the hierarchical nature of the superlattices, which offer control over the size, shape and composition of the QD building blocks as well as the geometry of the assembled superlattice. However, the myriad complex interactions at play during the assembly and oriented attachment processes lead to imperfections in epitaxial connections between QDs, which inhibit practical realization of the desired quantum phenomena. Local strain or defects in the connections reduce electronic coupling between QDs and lead to structural disorder which propagates through the film, effectively nullifying the picture of a miniband structure [2,3]. In order to attain control over the electronic properties, we must develop a deep understanding of the existing strain defects and how to avoid or mitigate them. Here, we present a method for visualizing and measuring distinct strain states in QDS and other quantum materials. Further, we assess the effect of mild annealing, which has been shown to improve electronic properties in QDS [4], on these strain states using in situ heating.

Strain has previously been mapped using geometric phase analysis (GPA) [5]. This technique, however, depends on identifying a fixed reference lattice. In our system - a periodic arrangement of crystalline QDs, where strain at the necks connecting neighboring QDs is of interest - a local reference frame is required to extract meaningful information. We, therefore, adapt a real space sine wave fitting approach, previously developed for scanning tunneling microscopy [6], to extract local lattice spacing $\left(q r^{-1}\right)$ and inplane orientation $(\theta)$, which are then mapped over the full field of view. Quantification of the spacing and orientation values allows us to distinguish between tensile and shear defects as detailed in Figs. $1 \mathrm{a}$ and $\mathrm{b}$. Figure 1a shows a tensile defect which appears clearly, as expected, in the spacing map for lattice fringes parallel to the epitaxial connection ( $\boldsymbol{a}$ direction), while the spacing map for the orthogonal direction and the two rotation maps are largely uniform. In the case of the shear defect (Fig. 1b), lattice fringes along the $\boldsymbol{b}$ direction exhibit large, localized rotation at the QD connection. By considering the local lattice spacing and orientation as opposed to tensile and shear strain directly, we also retain a direct visualization of the bending defects between QDs as shown in Fig. 1c.

Having developed a measurement method for tensile, shear and bending defects, we turn our attention to the evolution of such defects upon in situ heating. We use a MEMS based heating holder, which allows us to track a single region of interest through several heating cycles while maintaining atomic resolution over a large field of view. Figure 2 summarizes the effects observed for a QDS film before and after annealing for 50 minutes at $150-200^{\circ} \mathrm{C}$. We find that highly localized tensile and shear strains, examples of which are outlined by dashed and dotted lines respectively in the insets of Fig. 2, relax dramatically upon heating. However, QD-to-QD variation in the rotation maps, here called bending strain, remains largely unchanged.

Our observations of how different types of strain behave, and in particular relax, in response to heating provide insight into their relative energetics and suggest a route toward much sought after quantum phenomena in QDS. Furthermore, this strain analysis technique could find use in color center materials 
wherein local strain has a dramatic effect on the orbital splitting energies of the color centers such as NV defects in diamond. [7]
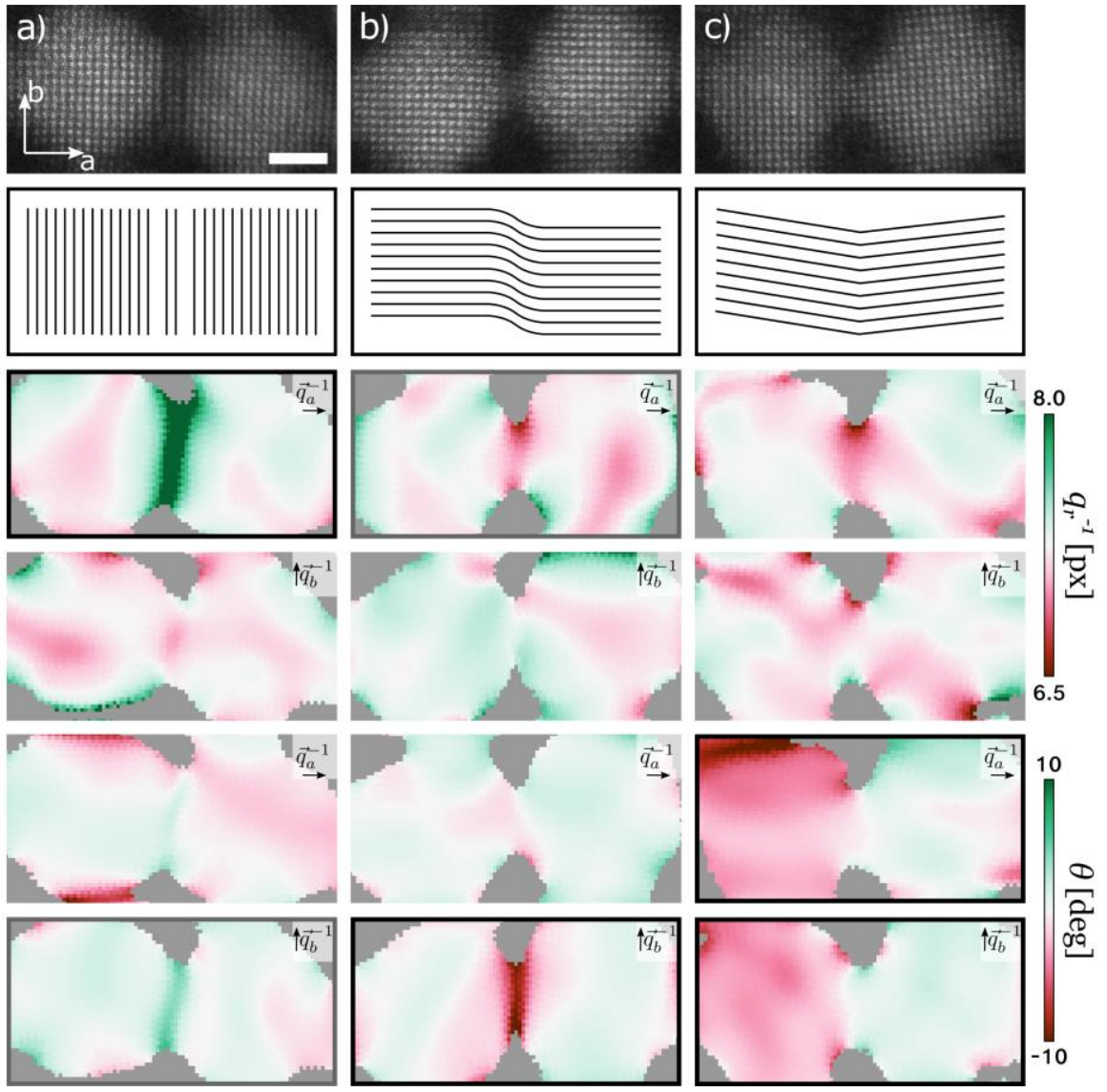

Figure 1. Examples of tension (a), shear (b), and bending (c) strain states in necks between QDs. From top to bottom each column shows a HAADF image of two QDs and the neck region between them, a schematic representation of the strain in the relevant lattice fringes, and maps of the spacing (qr-1) and orientation $(\theta)$ for the lattice vector indicated in the upper right corner of each map. The most characteristic map for each strain type is outlined in black, and complementary maps are outlined in gray. The scale bar is $2 \mathrm{~nm}$. 

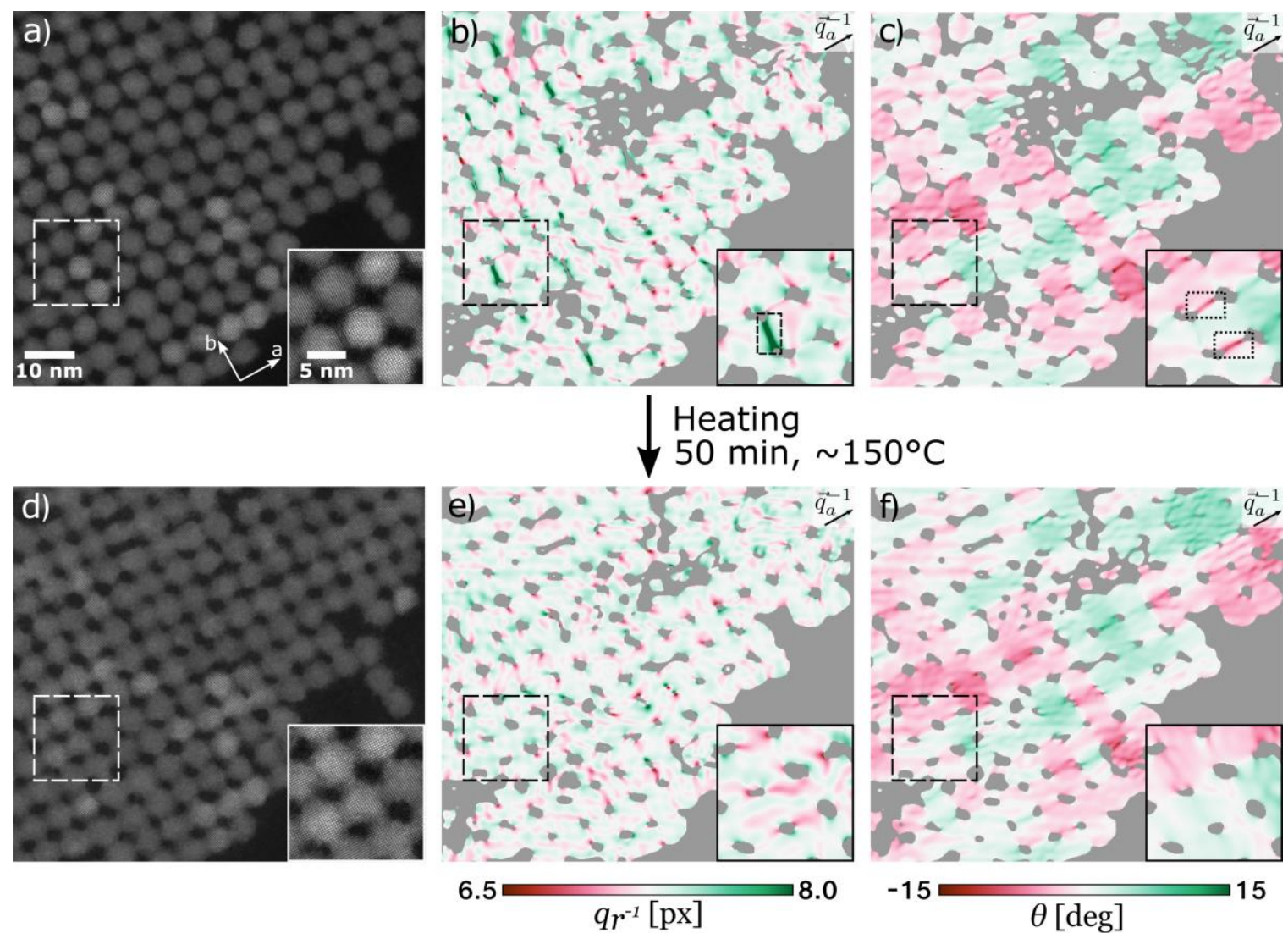

Figure 2. Evolution of strain states upon heating. (a,d) HAADF images of a region of QDS film before (a-c) and after (d-f) heating for 50 minutes at $150-200^{\circ} \mathrm{C}$, with corresponding spacing (b,e) an orientation $(\mathrm{c}, \mathrm{f})$ maps. The insets of $\mathrm{b}$ and $\mathrm{c}$ give examples of tensile and shear strain outlined by dashed and dotted lines, respectively. Upon heating, the strain disappears, as shown in the insets of e and $\mathrm{f}$.

\section{References}

[1] E. Kalesaki et al., Phys. Rev. X 4 (2014), p. 011010.

[2] B. Savitzky et al., Nano Lett. 16 (2016), p. 5714.

[3] C. R. Kagan and C. B. Murray, Nature Nanotech. 10 (2015), p. 1013.

[4] W. Walravens et al., ACS Nano. 13 (2019), p. 12774.

[5] M.J. Hÿtch, Scanning Microsc. 11 (1997), p. 53.

[6] Á. Pásztor et al., Phys. Rev. Res. 1 (2019), p. 033114.

[7] This work is supported by DOE (DE-SC0018026) and NSF (DMR-1719875, MRI-1429155, DGE1650441). 